\title{
Lean startup, Effectuation et Design Thinking : quel modèle combinatoire de management d'innovation dans les startups?
}

\author{
Miloud DAOUD ${ }^{1}$, Sara OUSGHIR ${ }^{2}$ \\ ${ }^{1}$ Laboratoire de recherches et d'études en Management, Entrepreneuriat et Finance ( \\ LAREMEF), Ecole National de Commerce et de Gestion, Fès, Maroc \\ ${ }^{2}$ Laboratoire de recherches et d'études en Management, Entrepreneuriat et Finance ( \\ LAREMEF), Ecole National de Commerce et de Gestion, Fès, Maroc
}

\begin{abstract}
Résumé : Les startups interviennent sur des marchés où l'incertitude est forte. Elles doivent par conséquence adapter en permanence leur Business Model (BM) aux besoins et exigences de leurs parties prenantes induisant l'adoption de méthodes de management plus réactif et flexible. Ce papier a pour objet de citer une revue de littérature concernant les différentes méthodes de management de l'innovation dans les entreprises en mode start-up dans l'objectif de développer un modèle combinatoire liant les trois approches : Lean Startup, Effectuation et Design Thinking. Ce modèle combinatoire représente le processus qui fait appel aux différentes approches de management d'innovation permettant de réussir la création, le développement et la croissance de projets innovants.
\end{abstract}

Mots-clés: Innovation, Business model, startup.

\begin{abstract}
The Business model, is a good tool to represente the history of startups and there evolutions, clients, ressources, revenus, value propositions, money angels, those elements can be easely managed by adapting the perfect methods of management that must therefore be flexible and responsive. This paper represents a literature review about the methods of management of innovation in start-up with the aim to develop a combinatorial model of the three methods: Lean Startup, Effectuation and Design Thinking.
\end{abstract}

Keywords: Innovation, Business model, startup.

\section{INTRODUCTION}

Dans un environnement comblé d'incertitude, les entreprises en mode startup se retrouvent dans un marché en pleine concurrence, leur souci est de réussir le développement et la mise en place de leurs innovations sur le marché. 
L'innovation est prédéfinie dans la littérature par le processus d'implantation de l'invention dans le marché. Ceci nécessite l'intégration de plusieurs éléments, entre autres : les ressources, les compétences, les partenaires, les outils et méthodes de management permettant de créer, proposer et partager de la valeur à partir d'un BM innovant.

Lean Startup, Design thinking et Effectuation, représentent les méthodes de management d'innovation permettant d'avoir une visualisation sur le processus de développement d'idée innovante en BM rentable.

\section{Un Panorama des TRAVAuX SUR LE Business MOdel : MOdeles ET OUTILS DE DEVELOPPEMENT}

Dans cette section, on vise à définir la notion du BM, de citer et d'analyser les différents changement et fluctuations possible dans les business model des startups innovantes.

\section{A. Business model, définitions conceptuelles}

Le Business Model (BM) a fait une vraie apparition pendant les années 90 avec la bulle d'internet. Lecoq et al. (2006), E. Moyon (2011) notent que « l'expression Business Model est utilisée de façon sporadique jusqu'au milieu des années $1970 »$. En effet, La croissance du nombre des start-ups implantées en 2000 a renforcé le développement des travaux contribuant à la consolidation du terme. C'est par exemple le cas des travaux d'Osterwalder et al. (2005) et ceux de Moyon (2011) qui ont éclairci le sens du concept à partir de ses deux mots composants : «Business » et « Model ». Selon ces auteurs, un « Business » ou « affaire » est « une activité de fourniture de biens et de services impliquant des aspects financiers, commerciaux et industriels ». Alors qu'un « Model » ou « modèle » est « une représentation simplifiée d'un processus ou d'un système ». Donc, on peut dire que les termes «Business Model » et « Model d'affaire », désignent la représentation simplifiée du processus d'affaires.

En effet, les académiques s'accordent sur un point : le BM représente les voies intentionnelles qu'une entreprise est susceptible d'emprunter et les choix qu'elle fait afin de créer de la valeur (Chesbrough, 2003 ; Chesbrough et Rosenbloom, 2002 ; Amit et Zott, 2001) et générer des revenus (Warnier et al. 2004 ; Chesbrough et Rosenbloom, 2002 ; Timmer, 1998). En effet, Xavier Lecocq met en avant la différence entre le BM et le model de revenue. Selon lui, le model de revenue est inclus dans le BM, il est le processus par lequel une entreprise va générer réellement l'argent. Alors que le $\mathrm{BM}$, comprend des éléments plus larges comme l'organisation interne ou externe, les compétences, les processus, etc.

\section{B. Méthodes, outils nécessaires pour développer un business model innovant}

Le BM est une modélisation, c'est-à-dire une représentation schématique de la logique de création, proposition et partage de la valeur...Quant au BM Canevas (BMC), il sert à décrire le modèle à partir de sa présentation structurée dans un canevas.

Le BMC est un outil développé par les deux chercheurs, Alexandre Osterwalder et Yves Pigneur (2010). Ces auteurs ont traduit les connaissances académiques sur le BM pour développer un outil simple et pratique à destination des managers et consultants. Ils proposent une matrice permettant d'élaborer un BM. Ce modèle compte neuf éléments appelés les neuf blocs de construction : Ressources clés, activités clés, alliances clés, structure de coûts, proposition de valeur, modèle de revenus, segmentation clients et canaux de distribution, relation client. 


\begin{tabular}{|c|c|c|c|c|}
\hline \multirow{2}{*}{ Partenaires Clés } & Activités Clés & \multirow{2}{*}{$\begin{array}{l}\text { Proposition de } \\
\text { valeur }\end{array}$} & $\begin{array}{c}\text { Relation avec le } \\
\text { client }\end{array}$ & \multirow{2}{*}{$\begin{array}{l}\text { Segments } \\
\text { clients }\end{array}$} \\
\hline & Ressources Clés & & $\begin{array}{l}\text { Moyen de } \\
\text { distribution }\end{array}$ & \\
\hline \multicolumn{2}{|c|}{ Structure des Coûts } & & \multicolumn{2}{|c|}{ Flux de revenus } \\
\hline
\end{tabular}

Figure 1: la matrice du Business Model, Osterwalder et Pigneur (2010).

Sur le plan théorique, le concept de Business Model (BM) s'est développé en intégrant deux approches complémentaires permettant d'articuler le concept avec celui des capacités dynamiques : l'approche contenu et l'approche processus.

- L'approche contenu du BM : L'approche contenu permet d'identifier les éléments de contenu du BM et leurs évolutions dans le temps (E. Moyon, 2011 ; X. Lecocq et al., 2006 ; D. Raff, 2000). D'après X. Lecocq et al. (2006), le BM est un système soumis à un changement continuel impliquant des changements de certaines de ses composantes. Ces changements peuvent être délibères, actionnés par des décisions managériales, ou relevant de la dynamique interne du BM.

L'approche contenu permet de prendre en compte les transformations des composantes du BM d'une période à une autre. J. Linder et S. Cantrell (2000) proposent un modèle de changement de BM à quatre paliers, suivant le degré de changement de la logique du BM et le nombre de composantes impactées par le changement.

« Realization model »: Dans ce cas, Le changement impacte uniquement l'organisation.

« Renewal model »: Le changement impact l'organisation et le modèle de revenu, le changement est qualifié de faible.

«Extension model »: Il représente un changement important qui touche à la fois l'organisation, le modèle de revenu et la proposition de valeur.

« Journey model » : Le changement impacte l'ensemble des composantes, le changement est radical impliquant une nouvelle logique du BM.

A. Osterwalder et Y. Pigneur ont parlé, à ce titre, d'épicentres de l'innovation qui représente le résultat du changement du BM à partir de la modification d'une des blocs du BMC. Ce dernier peut être utilisé pour concevoir de nouveaux BM ou reconfigurer des modèles existants. La décomposition du BM en blocs étant au cœur de l'outil. A.Osterwalder et Y.Pigneur identifient quatre épicentres de l'innovation de BM. 


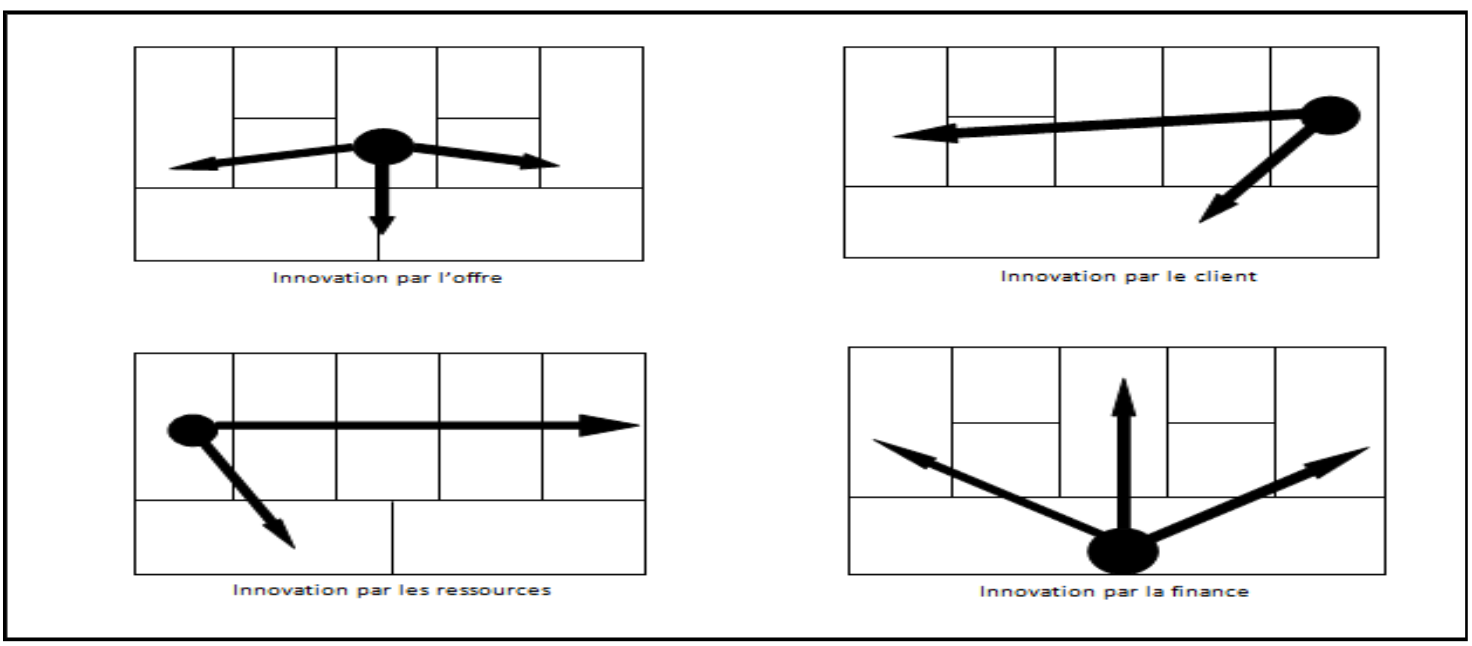

Figure 2: les épicentres de l'innovation du Business Model (Osterwalder et pigneur, 2010)

- L'innovation par l'offre: Il s'agit pour l'entreprise innovante de formuler des propositions de valeur innovantes à partir desquelles le reste du modèle est conçu. Dans ce cas, les entreprises repensent profondément le modèle économique pour réaliser et tirer profit de proposition de valeur innovante.

- L'innovation par le client: Elle consiste à servir des segments de clients non encore atteint ou à améliorer significativement l'accès de certains clients aux offres existantes. Là encore tous les blocs du BM ont dû être repensés pour rendre possible le service de ce segment de clientèle mais le point de départ du nouveau business est le client.

- L'innovation par les ressources: Elle désigne le renouvellement du BM à partir des infrastructures et des partenariats développés par l'entreprise. Lorsque l'entrepreneur choisit d'exploiter au maximum ses ressources, l'entreprise « ouvre» son BM pour valoriser ses actifs ou donner accès à ses facilités de production pour en augmenter la rentabilité.

- L'innovation par la finance: Elle consiste à dessiner un nouveau modèle à partir de mécanismes de monétisation nouveau. La réduction de la structure de coût est également un point de départ pour repenser des modèles.

- L'approche processus du BM: L'approche processus (O. Ammar, 2010; A.G. Pateli et G.M. Giaglis, 2005) fonde son analyse sur les mécanismes permettant d'expliquer les phases de changement du BM. A.G. Pateli et G.M. Giaglis (2005) proposent un modèle de changement processuel à trois phases. La première phase consiste à faire un diagnostic approfondi du BM initial. La deuxième phase est celle de l'identification de l'influence technologique. La troisième est celle du changement. Elle comporte trois étapes : tout d'abord, définir les différents scénarios, puis décrire les nouveaux BM (acteurs, possibilités du marché, modèle de relations, modèle de revenu, facteurs de succès) et enfin évaluer l'impact du changement.

L'approche processus met en avant le rôle des facteurs internes et externes dans le changement du BM. Parmi ces facteurs, les capacités dynamiques de l'entreprise (D.J. Teece et al., 1997). Celles-ci reflètent la capacité d'une entreprise à intégrer, construire et reconfigurer ses ressources et compétences internes et externes, pour atteindre des formes nouvelles et innovantes plus compétitives dans des environnements turbulents et dynamiques (D.J. Teece et al., 1997). 
L'activité de design d'un BM, développé par J. Magretta (2002), a pour objet de procéder à la mise en place de la méthode expérimentale. En effet, le design de BM consiste pour l'entrepreneur à formuler un premier BM, c'est-à-dire un scénario cohérent de création, proposition et partage de la valeur. En reprenant le $\mathrm{BMC}$, cela revient à remplir les neuf blocs et à être capable d'expliciter la logique qui tient ensemble ces éléments. Le test d'hypothèses au niveau des différentes composantes du BM permet de d'améliorer l'idée du projet initial. Plus on test plus on raffine et on reformule le BM innovant. Le pivot, est l'action qui permet de passer vers BM2 radicalement diffèrent du BM1.

\section{ELABORATION DES BM INNOVANTS : ESSAI D'UN MODELE INTEGRATEUR}

Cette section a pour objet d'expliquer les méthodes utilisées par les startups pour développer un BM innovant. En se basant sur ces dernières nous proposons un nouveau modèle intégrateur des trois méthodes d'innovation : Effectuation, Design thinking et Lean startup

\section{A. L'effectuation}

L'effectuation est une approche managériale développée dans les années 90 par Saras Sarasvathy. Cette approche stipule que le point de départ du projet est l'entrepreneur, avec sa personnalité, sa connaissance et ses relations, qui constituent ses moyens de base. Avec cela, l'entrepreneur détermine ce qu'il peut faire (les moyens déterminent les buts possibles). Si tout se passe bien, l'action entraîne l'engagement de nouvelles parties prenantes, qui du coup apportent de nouveaux moyens au projet. Ces nouveaux moyens permettent à l'entrepreneur de définir de nouveaux buts, plus ambitieux, et de reboucler sur le point de départ : nouveaux moyens, nouveaux buts, qui signifient nouvelles actions et nouvelles parties prenantes, qui signifient moyens supplémentaires, etc. Avec cette démarche, l'opportunité se construit au fur et à mesure au lieu d'être découverte à travers un éclair de génie. Le processus entrepreneurial est ainsi résumé dans la fig.4.

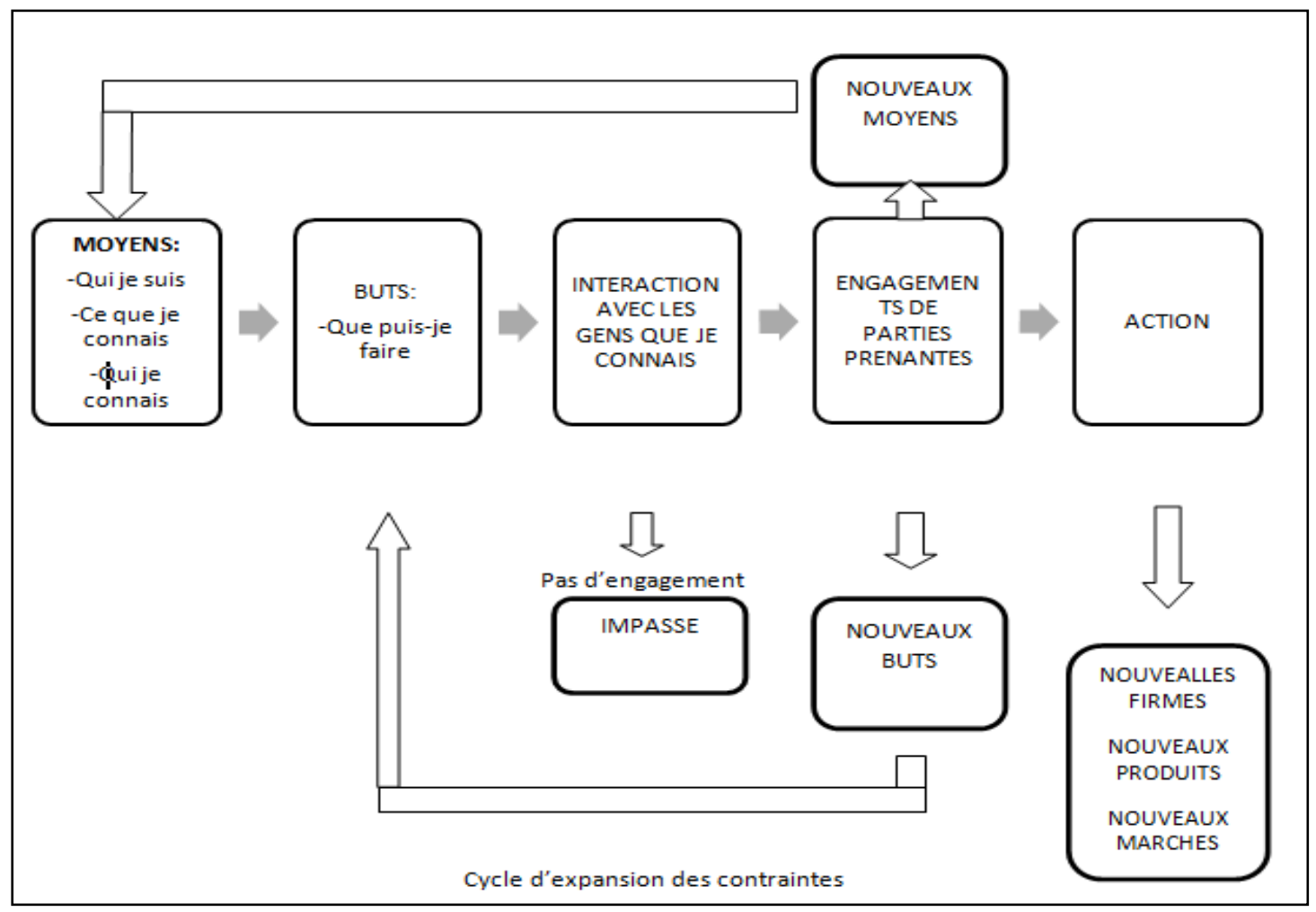

Figure 4 : Le processus entrepreneurial selon le modèle effectual (Philippe Silberzahn, 2013) 
L'effectuation constitue une approche dynamique dans laquelle les buts de l'entrepreneur émergent à partir de ses moyens disponibles (son identité, ses connaissances et son réseau...). L'entrepreneur imagine une série de couples produits-marchés possibles, qu'il expérimente afin de déterminer leur potentiel. En fixant un niveau de pertes acceptables, il peut développer l'offre et la demande avec un minimum de ressources, en contrôlant les risques d'échec. En effet, Le raisonnement effectuale est non déterministe et émergent; il s'oppose en cela à l'approche dite « Causale » qui fixe des buts clairs et stables dans le temps et qui correspond à une approche délibérée de la stratégie. (Philippe Silberzahn, 2013).

La logique causale, largement inspirée des théories du choix rationnel (Perry et al.; 2011, Corner et Ho; 2010), permet la sélection optimale de ressources nécessaires pour atteindre un but préalable (Sarasvathy; 2001). qui, dans le cadre de la création d'entreprise, peut se définir comme le désir de créer une entreprise à partir d'un couple produit-marché prédéfini.

Cette approche met l'accent sur l'importance de la prévision et de l'analyse préalable pour éviter les mauvaises surprises. Elle fonctionne bien dans les marchés établis, dans lesquels les structures, les concepts produits et marché sont connus. Elle est particulièrement indiquée dans les cas de relative prévisibilité du couple produit-marché, lorsque l'offre et/ou la demande préexiste (Sarasvathy et al., 2003).

\section{B. Lean startup}

La méthode Lean Startup (Ries E., 2011) est l'un des mouvements de conception les plus populaires actuellement. Elle repose essentiellement sur des boucles de Feed-Back. Le processus ne se présente donc pas avec un début et une fin mais s'inscrit dans un cycle continu débutant avec un Minimum Viable Produit (MVP1) qui devra proposer une valeur suffisante pour intéresser le client final. Le MVP a pour objet de valider ou pas l'hypothèse de valeur définie dans le couple produit/projet.

Cette approche repose sur un processus itératif court, centré sur l'expérimentation, et structuré par trois opérations : Construire, Mesurer et Apprendre (Fig.5).

CONSTRUIRE : Le prototype est essentiel car il permet d'organiser des interactions tangibles avec les clients potentiels bien avant d'avoir développé le produit final. Dans le jargon Lean startup, on parle de MVP. Chaque MVP est spécifique à un cycle d'apprentissage. Il est donc conçu dans le but de valider quelques hypothèses précises et non toutes à la fois. Cette approche permet de maximiser la précision du test, réduire son coût et gagner du temps. (Alexandre Terseleer et Olivier Witmeur, 2013).

MESURER : Cette phase de mesure consiste à collecter des données reçus suite à l'utilisation du MVP par les utilisateurs. L'analyse des feed-back permet de valider ou non les hypothèses, et décider quelle direction suivre pour la suite afin d'atteindre, après plusieurs cycles, une bonne adéquation entre le produit et le marché (Alexandre Terseleer et Olivier Witmeur, 2013).

APPRENDRE: Le troisième principe tient à l'adoption d'une dynamique d'apprentissage par itérations successives et rapides. Afin d'acquérir une bonne connaissance du consommateur, Lean Startup met en place une succession de cycles d'interactions avec les clients, où chaque cycle doit permettre à l'entrepreneur de tester

\footnotetext{
${ }^{1}$ Le MVP est la plus petite unité testable par des utilisateurs potentiels et la moins élaborée, pour exemple, une maquette ou un prototype. Le MVP a vocation à être testé le plus rapidement possible de sorte à mesurer ses effets et à collecter des données.
} 
une hypothèse grâce à l'expérimentation, de collecter les données et d'en tirer des conclusions. (Alexandre Terseleer et Olivier Witmeur, 2013)

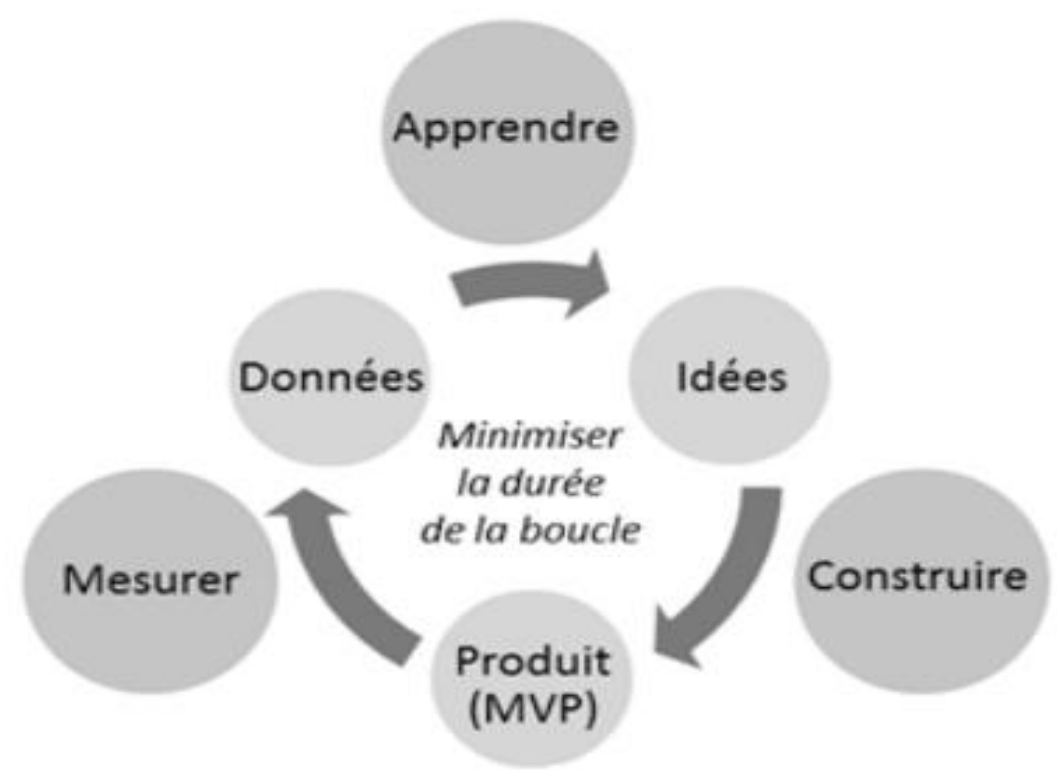

Figure 5 : Le processus Lean Startup (Ries E., 2011)

Le risque majeur à éviter est de passer trop de temps sur la phase Construire, et de retarder ainsi les phases Mesurer et Apprendre. En effet l'objectif doit toujours être de confirmer le plus rapidement possible si l'idée et le produit créent de la valeur pour le client, et d'apprendre quels sont les points faibles et les points forts de cette idée. De nombreuses startups passent des mois, voire des années à peaufiner un produit sans jamais le confronter aux utilisateurs. Lorsqu'ils apprennent finalement que ceux-ci ne sont pas intéressés par leur idée, il est souvent trop tard et la startup meurt.

En adoptant l'approche Lean, les entrepreneurs développent leurs innnovation de manière itérative. Ils n'adoptent donc pas un modèle classique " phase de conception, puis phase d'exécution », mais une série d'itérations conception/mise en oeuvre grace à l'interaction avec son environnement.

Spécifiquement, avec lean, l'interaction se fait avec les premiers clients. L'hypothèse est qu'il est impossible de savoir a priori ce que veulent ces derniers. La solution réside dans le « faire » : construire le produit, le mettre dans les mains des clients et mesurer leur réaction. Puis tirer les leçons de cette réaction, et utiliser cet apprentissage pour remettre le produit sur l'établi et l'améliorer. D'où le cycle « construire-mesurerapprendre » de lean. L'interaction avec l'extérieur est donc fondamentale, et lean met bien en avant la nécessité de « sortir de chez soi », d'aller au contact de son marché potentiel par le développement d'une première offre qu'on améliore ensuite.

L'effectuation met elle aussi en avant l'interaction avec l'environnement extérieur comme base fondamentale de la progression du projet entrepreneurial, mais elle le fait de manière plus générale. L'effectuation, elle, ne s'intéresse pas spécifiquement au produit, mais au projet dans son ensemble, et à la démarche de l'entrepreneur pour développer celui-ci.

Alors que lean startup est surtout une méthode de développement de nouveaux produits, et ne dit pas grandchose sur les autres aspects du projet entrepreneurial. 
La mise en point de la relation entre les deux approches : lean startup et effectuation, nous permet de proposer un schéma intégrant leurs principes. En effet, lean startup, en tant que approche intervenant au niveau de développement du produit innovant, nous proposons de l'intégrer au niveau d'une des étapes du processus effectual qui a pour objet la détermination de l'objectif à partie des moyens existant, ces objectif représente des idées à tester sur le terrain grâce à l'interaction de parties prenantes vis-à-vis le MVP.

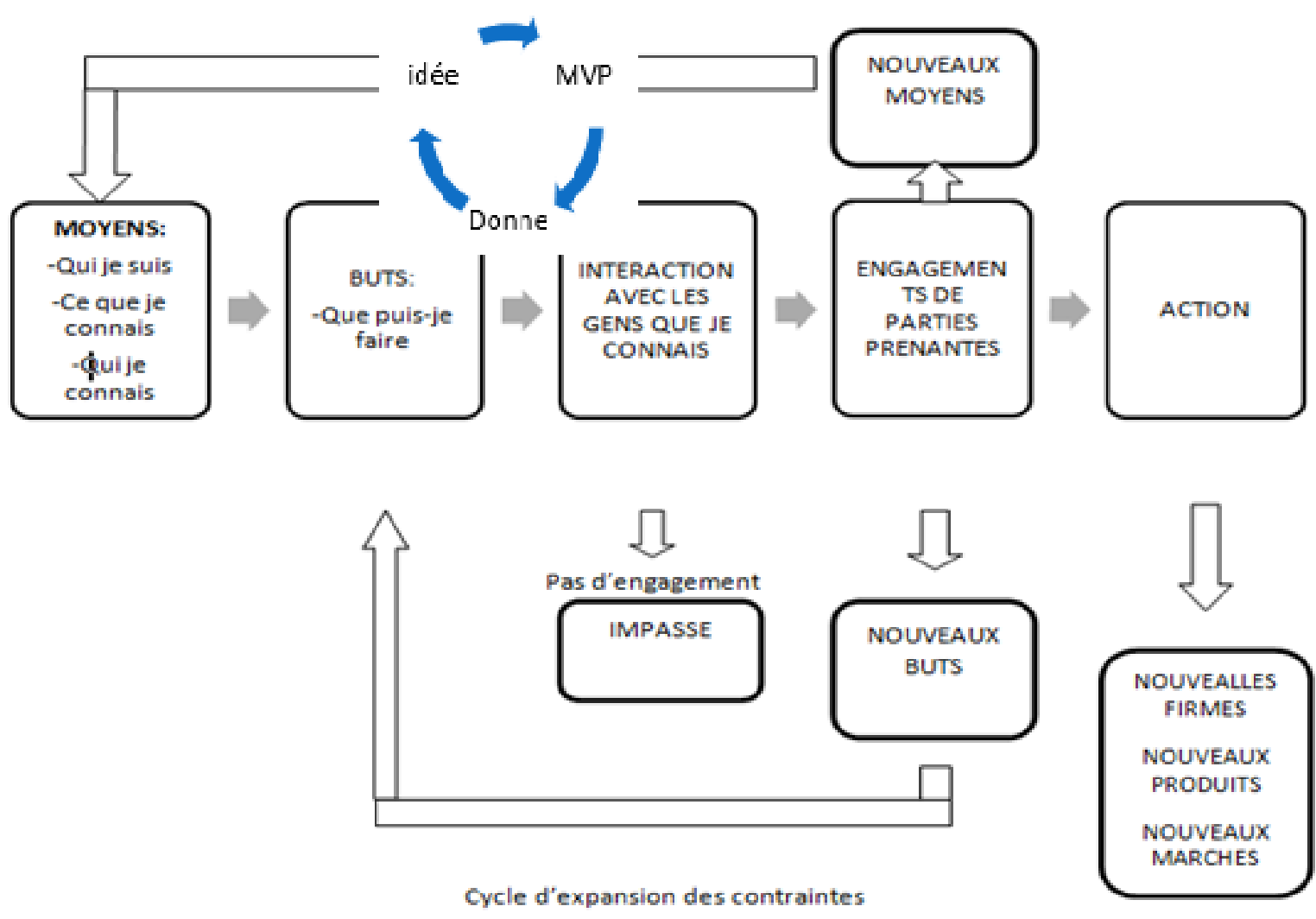

Figure 6 : Combinaison des deux processus d'innovation : Effectuation et Lean Starup .

\section{Le design thinking}

La définition « officielle» du design thinking est donnée par Tim Brown, P.D.G. d'IDEO : « Le design thinking est une discipline qui utilise la sensibilité, les outils et méthodes des designers pour permettre à des équipes multidisciplinaires d'innover en mettant en correspondance attentes des utilisateurs, faisabilité technologique et viabilité économique »

Au sein de la d.school Paris, le processus d'innovation par le Design Thinking de référence est celui défini par Tim Brown dans Harvard Business Review en 2009, qui distingue trois phases : inspiration, idéation, implémentation. 


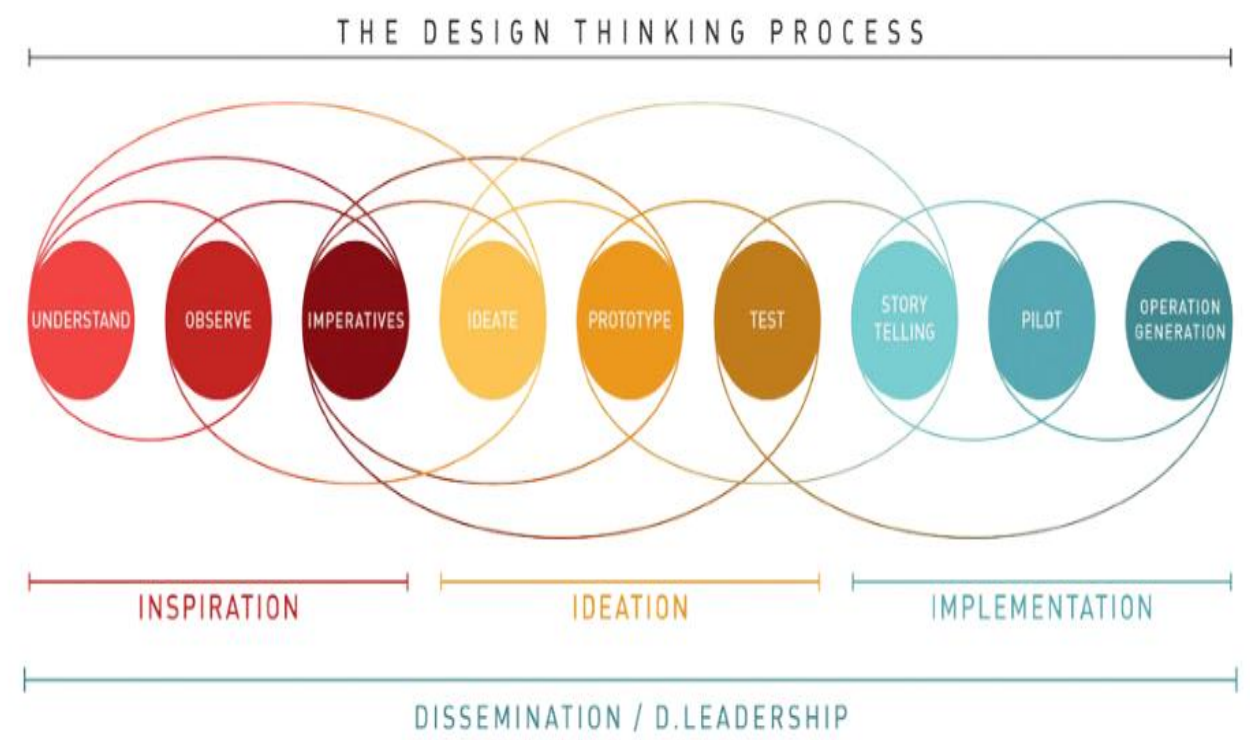

Figure 7: Processus de design thinking (Tim Brown, 2009)

\section{1) Phase d'inspiration}

Cette phase a pour principale mission de comprendre le comportement du consommateur potentiel en se basant sur les résultats de la recherche ethnographique. Cette recherche a pour objectif de déterminer les besoins latents des utilisateurs.

\section{2) Phase d'idéalisation}

«L'idéation est le processus de générer, développer et tester des idées qui peuvent aboutir en des solutions

» (Tim Brown, 2008). L'objectif dans cette phase est de confronter rapidement les idées avec des utilisateurs pour percevoir leurs intérêts en évitant le gaspillage en temps et argent.

\section{3) Phase d'implémentions}

L'implémentation regroupe toutes les activités nécessaires pour transformer le projet de l'état de conception à une réalité. Elle consiste à transformer l'idée en prototype industriel, en contactant les soustraitants pour les parties non réalisables en interne, de mettre en avant des difficultés d'industrialisation et de construire un produit le plus proche du produit final pour valider son usage.

Le processus d'innovation par le design thinking ne commence pas par l'émission d'idées, mais par la compréhension de ceux pour qui on veut innover. Les clients représentent l'unique source d'inspiration pour tout processus de design thinking.

Margaret Mead, anthropologue du XXe siècle, soulignait qu'il y a une différence entre ce que disent les gens, ce qu'ils font et ce qu'ils disent qu'ils font. ... C'est pourquoi il faut prendre en considération la posture de l'anthropologue dans la détermination des besoins des clients. Comme le confirme Tom Kelley, il ne faut pas juger mais développer une empathie authentique et un amour vrai d'observer et de comprendre l'autre. L'objectif est de gagner en pertinence par rapport à ceux pour qui on veut innover, afin de « frapper juste » : juste en pertinence dans l'identification des besoins réels, et juste en quantité dans la compréhension de leur hiérarchie.

Dans le tableau ci-dessous, nous avons repris les travaux de comparaison entre les deux méthodes de management d'innovation : «Lean Startup » et «Effectuation », développé par Philippe Silberzahn (2014), en rajoutant une colonne indiquant la méthode « design thinking », ainsi que des lignes permettant d'avoir plus de 
critères de comparaison entre les trois méthodes, à savoir : Outils de changement du BM, processus de développement, produit ou projet, type d'innovation.

Tableau I: Comparaison entre les trois méthodes de management d'innovation,

\begin{tabular}{|c|c|c|c|}
\hline & Lean Startup & Effectuation & Design thinking \\
\hline $\begin{array}{l}\text { Nature } \quad \mathrm{du} \\
\text { problème }\end{array}$ & $\begin{array}{l}\text { Découvrir le besoin } \\
\text { client }\end{array}$ & Résoudre l'incertitude & $\begin{array}{l}\text { déterminer les besoins réels } \\
\text { des utilisateurs d'une façon } \\
\text { empathique }\end{array}$ \\
\hline Unité d'analyse & MVP & $\begin{array}{l}\text { Le réseau de valeur en } \\
\text { construction }\end{array}$ & Prototype \\
\hline Paradigme & $\begin{array}{l}\text { Apprentissage } \\
\text { l'environnement }\end{array}$ & $\begin{array}{l}\text { Transformation } \\
\text { d'environnement }\end{array}$ & $\begin{array}{ll}\text { Compréhension } & \text { de } \\
\text { l'environnement } & \end{array}$ \\
\hline $\begin{array}{ll}\text { Base } & \mathrm{de} \\
\text { progression } & \end{array}$ & Itération produit & $\begin{array}{l}\text { Engagement des parties } \\
\text { prenantes dans le projet }\end{array}$ & $\begin{array}{l}\text { Adéquation produit/attente } \\
\text { du client }\end{array}$ \\
\hline $\begin{array}{lll}\begin{array}{l}\text { Objectif de la } \\
\text { méthode }\end{array} & \\
\end{array}$ & Vitesse & Viabilité & $\begin{array}{ll}\text { Viabilité, } & \text { Faisabilité, } \\
\text { désirabilité } & \\
\end{array}$ \\
\hline $\begin{array}{l}\text { Rôle des parties } \\
\text { prenantes autres } \\
\text { que clients }\end{array}$ & Aucun & Fondamental & $\begin{array}{l}\text { Aident dans l'analyse des } \\
\text { besoins latents des } \\
\text { utilisateurs potentiels }\end{array}$ \\
\hline $\begin{array}{l}\text { Critères de } \\
\text { réussite } \\
\text { entrepreneuriale }\end{array}$ & $\begin{array}{l}\text { Produit répondant aux } \\
\text { besoins de clients } \\
\text { identifiés }\end{array}$ & $\begin{array}{l}\text { Création d'un artefact social, } \\
\text { produit, entreprise, marché }\end{array}$ & $\begin{array}{l}\text { L'amélioration de } \\
\text { l'expérience utilisateur ou la } \\
\text { création d'une offre } \\
\text { radicalement nouvelle }\end{array}$ \\
\hline $\begin{array}{ll}\text { Outils } & \text { de } \\
\text { Changement } & d u \\
\text { BM } & \end{array}$ & $\begin{array}{l}\text {-Pivots jusqu'à atteindre } \\
\text { l'adéquation } \\
\text { produit/marché } \\
\text { - Interactions avec le } \\
\text { client } \\
\text {-itérations du processus }\end{array}$ & $\begin{array}{l}\text { Interactions avec } \\
\text { l'environnement extérieur: } \\
\text {-engagement+co-construction } \\
\text {-Les parties prenantes apportent } \\
\text { de nouveaux moyens et par } \\
\text { conséquence de nouveaux buts } \\
\text {-itération pour objet de faire } \\
\text { face à l'incertitude }\end{array}$ & $\begin{array}{l}\text { - Interaction ethnographique } \\
\text { avec le client }\end{array}$ \\
\hline $\begin{array}{l}\text { Processus de } \\
\text { développement }\end{array}$ & $\begin{array}{l}\text { Construire->Mesurer- } \\
\text { >Apprendre }\end{array}$ & $\begin{array}{l}\text { Moyens->But->Interaction } \\
\text { avec les gens que je connais-> } \\
\text { Engagement des PP->Action }\end{array}$ & $\begin{array}{l}\text { Inspiration->Idéalisation- } \\
\text { >Implémentation }\end{array}$ \\
\hline Produit ou projet & Développent du produit & Développement projet & $\begin{array}{l}\text { Développent du produit et } \\
\text { marché }\end{array}$ \\
\hline Type d'innovation & Plus Incrémentale & Plus Radical & Radical\&Incrémentale \\
\hline
\end{tabular}

Source: adapté de Philippe Silberzahn (2014)

Le processus d'innovation par le design thinking se base essentiellement sur les pratiques de la recherche ethnographique, qui ont pour objectif d'analyser en profondeur les attentes des clients autant que principale partie prenante. De ce fait, on peut revoir le processus précédemment développé en indiquant les clients 
comment principale partie prenante et en rajoutant la recherche ethnographique comme élément principale dans le processus d'innovation.

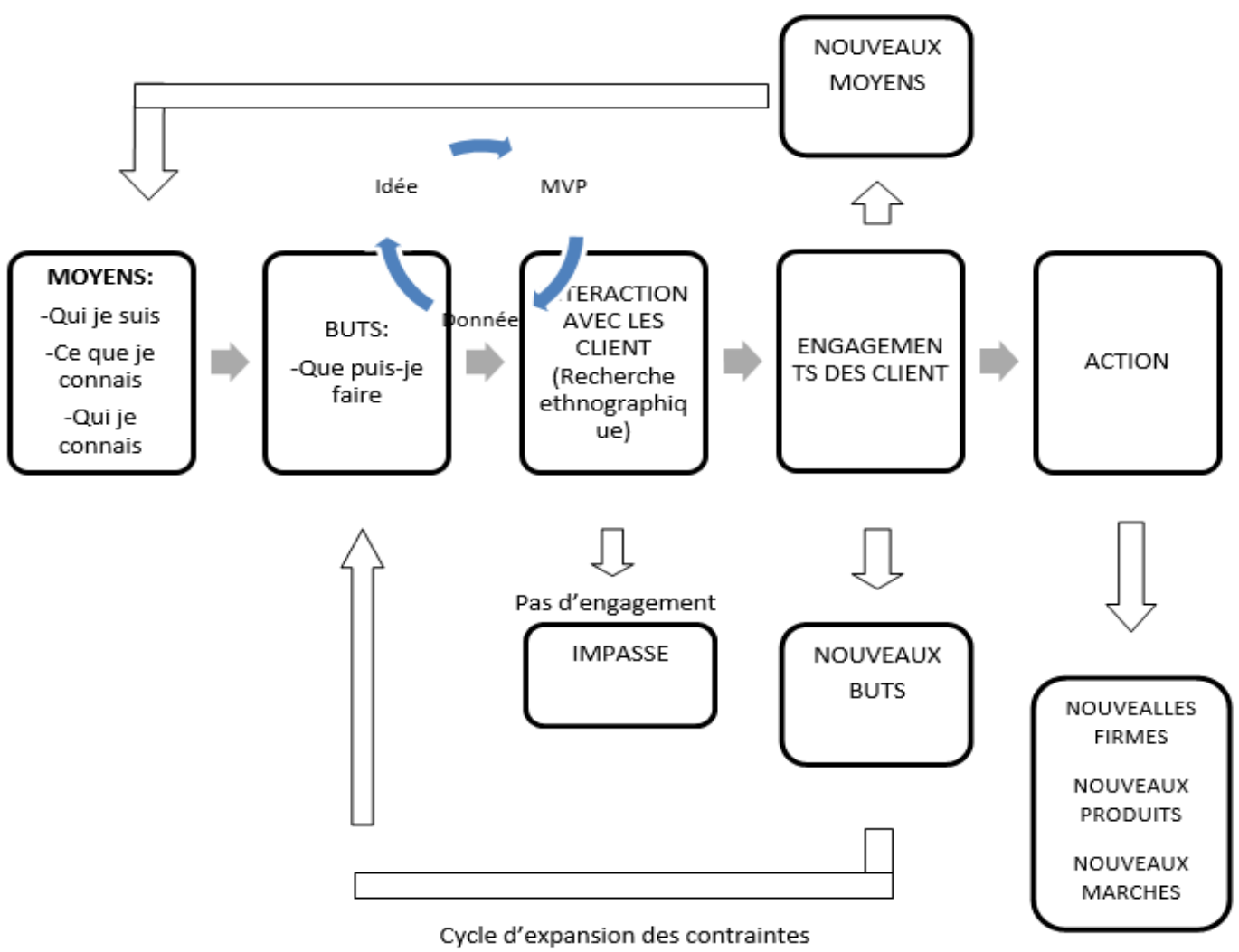

Figure 8 : Combinaison des trois processus d'innovation : Effectuation, Lean Startup et Design thinking

Ce processus d'innovation permet de revoir les différentes méthodes précédemment cités d'une maniére intégrative, ça intègre à la fois le processus d'innovation par l'effectuation et ses principes : commencer par les moyens existants pour déterminer les buts à attendre, qui s'améliorent par l'intégration de nouvelle partie prenante.

Ce processus intègre également le processus Lean Startup, Par le principe de faire tester les idées permettant de développer les MVP grâce, entre autres, l'interaction des parties prenantes.

En rajoutant les principes du design thinking, qui a pour objet d'étudier en profondeur le comportement des consommateurs en appliquant la recherche ethnographique en traitant les clients comme la principale partie prenante. De ce fait, On peut revoir les différentes étapes du processus en intégrant les deux éléments : 1-Les clients, 2-Recherche ethnographique.

1- Les clients : L'interaction avec le MVP se fait sur la base des attentes, besoins et avis des clients,

2- La recherche ethnographique : permet d'étudier le comportement et la manière avec laquelle interagissent les clients avec MPV

\section{CONCLUSION}

Il n'existe pas de cadre qui prédéfinisse l'application d'une démarche permettant d'intégrer les trois méthodes d'innovation. La complémentarité des approches favorise l'innovation dans son intégralité, et ce tout au long du processus de développement du BM. 
Créer une startup, c'est innover dans chacune des phases du projet, de la phase d'idéation à la phase de lancement. L'innovation se doit d'être une démarche holistique et conceptualisée. Certaines phases du développement d'innovation se prêtent davantage à l'application d'une méthode précise d'innovation.

En réalité, les trois méthodes favorisent l'innovation à chaque phase de développement. Elles sont des réponses innovantes à des problématiques précises, il est donc essentiel de les combiner afin de bénéficier de leur complémentarité.

De ce fait, nous avons proposé un modèle intégrateur des trois approches d'innovation. En effet, Au cours du processus de création du BM, les startups peuvent intégrer les trois méthodes de management d'innovation afin de réussir au mieux leurs projets.

Les conditions d'adoption du Design Thinking, du Lean Startup et de l'effectuation ne sont pas toujours optimales, voire parfois en contradiction avec les objectifs poursuivis. Elles doivent être adaptées au domaine d'activité, à la culture, au type d'innovation adopté par la startup. De ce fait, on peut dire que ce n'est pas l'utilisation mais bien l'application contextualité de ces approches innovantes qui garantit la réussite de la startup.

\section{BIBLIOGRAPHIE}

[1] Ammar, "Adaptation et mise en place d'un processus d'innovation et de conception au sein d'une PME", Thèse de doctorat en Sciences des Métiers de l'Ingénieur, Sous-direction de Mr J. Nadeau, ENSAM, Paris, 2011;

[2] Ben Romdhane, "capacités dynamiques et changement du business model : le cas Keyrus Equinoxes Tunisie", La Revue des Sciences de Gestion, Vol 2, N 278-279, 2016, pp. 141-149

[3] O. Brette et al., "Management de l'innovation et business models des PMI. Le cas de l'industrie plasturgiste", Revue Management \& Avenir, Vol.1 N 41, 2011, pp. 236-253;

[4] S. Brosia, "Management stratégique de Start up innovantes et création de valeurs", Thèse de doctorat en gestion, Sous-direction de Mme Line BERGERY, Université de France, 2017 ;

[5] P. CHAIX, “innovation et entrepreneuriat", E-thèque, France, 2015 ;

[6] d'Andria , "un éclairage sur le processus entrepreneurial des mampreneurs. Étude exploratoire de leur dynamique effectuale", Revue de l'Entrepreneuriat, Vol. 13, 2014, pp. 11 à 33 ;

[7] D.FranceDauchy "“7 étapes pour un business model solide : réinventer la création de valeur avec méthode”,DUNOD, France, 2010 ;

[8] Fonrouge, "Entrepreneuriat et innovations organisationnelles. Pratiques et principes", Revue française de gestion, Vol.5, $\mathrm{N}^{\circ}$ 185, , 2012, pp. 107-123;

[9] Gay, L. Szostak, “Management de l'innovation”, DUNOD, France, 2017

[10] M. Haggege, "Les raisonnements à l'œuvre dans la conception de business models innovants", Thèse de doctorat en gestion, Sous-direction de Mr V.Chanal, Ecole doctorale de science de gestion de Grenoble, 2013;

[11] A. Iselin, “ Proposition de valeur et positionnement prix la place de la valeur client dans le pricing d'offres innovantes : le cas des start-up technologiques", Thèse de doctorat en gestion, Sous-direction de Mr. A. Bloch, CNAM, 2009 ;

[12] M. Jungerman, “Création d'entreprise : la stratégie du bon sens". AFNOR, 2014;

[13] B.Laperche, G. Lefebvre, "stratégie environnementale, innovation et mutation des firmes”, « Innovations », vol 1 N³7, 2012 , pp.127-154;

[14] R.Lasch, H.Le Delacour et S. Liarte (2014), “Management stratégique de l'innovation”, Edition Economica, France, 2014 ;

[15] Léger-Jarniou, "Réussir son étude de marché Pour construire son business model”, DUNOD, France, 2016 ;

[16] L.Lehmann-Ortega H. Musikas et J. Schoettl, “ (Ré) inventez votre Business Model”, DUNOD, France, 2017 ; 
[17] Leon, "La construction des Business Models des fournisseurs de services d'infrastructure Cloud Computing (IaaS)", Thèse de doctorat en gestion, Sous-direction de Mme. M. Martin, Universite de Nice Sophia Antipolis, 2015;

[18] Mathieu et V. Hillen, "le design thinking par la pratique", EYROLLES, France, 2018 ;

[19] E. Ries, "Lean startup", Pearson, France, 2016;

[20] Maurya, "Scaling Lean : Maîtriser les indicateurs-clés de la croissance de votre start-up”, Diateino, France, 2014 ;

[21] J. Péché, F. Mieyeville et R. Gaultier, "Design thinking : le design en tant que management de projet”, Entreprendre \& Innover, Vol.1, $\mathrm{N}^{\circ} 28,2016$, pp 83-94;

[22] J. Roux, "Ca va marcher ! 7 étapes pour entreprise profitable. Business model, mode d'emploi”, EYROLLES, France, 2012 ;

[23] F.Roy et S.Yam, "Les déterminants de la survie et de la croissance des start-up TIC". Revue française de gestion, Vol.2, N 155 , 2005, pp. 37-50;

[24] P. Silberzahn, “Effectuation: Les principes de l'entrepreneuriat pour tous”, PEARSON, France, 2015 ;

[25] Tellier, "Bonnes vibrations : Quand les disques mythiques nous éclairent sur les défis de l'innovation", EMS Editions, France, 2017 ;

[26] Terseleer et, O. Witmeur, "lean startup : mode ou nouvelle bonne pratique ?", « Entreprendre \& Innover », vol 3, N 19, 2013, pp. 21 à 28 ;

[27] Valérie, "Business Models dans l'innovation, pratiques et methodes". Presses Universitaires de Grenoble, France, 2012 ;

[28] T. Verstraete, "Une théorie conventionnaliste du Business Model en contexte de création d'entreprise pour comprendre l'impulsion organisationnelle", Communication au congrès CIFEPME, Université Bordeau IV, 2014;

[29] S. Vincent, "veille stratégique en gestion de l'innovation : concepts et enjeux", Revue internationale d'intelligence économique, Vol. $9 \mathrm{~N}^{\circ} 53,2017$, pp. 53 à 67 ;

[30] O. Witmeur et P. Silberzahn, "nouveaux outils, nouvelles pratiques : effets de mode ou changements profonds ?", Revue Entreprendre \& Innover, vol3, N 19, 2013, pp 7 à 8, ; 